\title{
Internationale Studienergebnisse
}

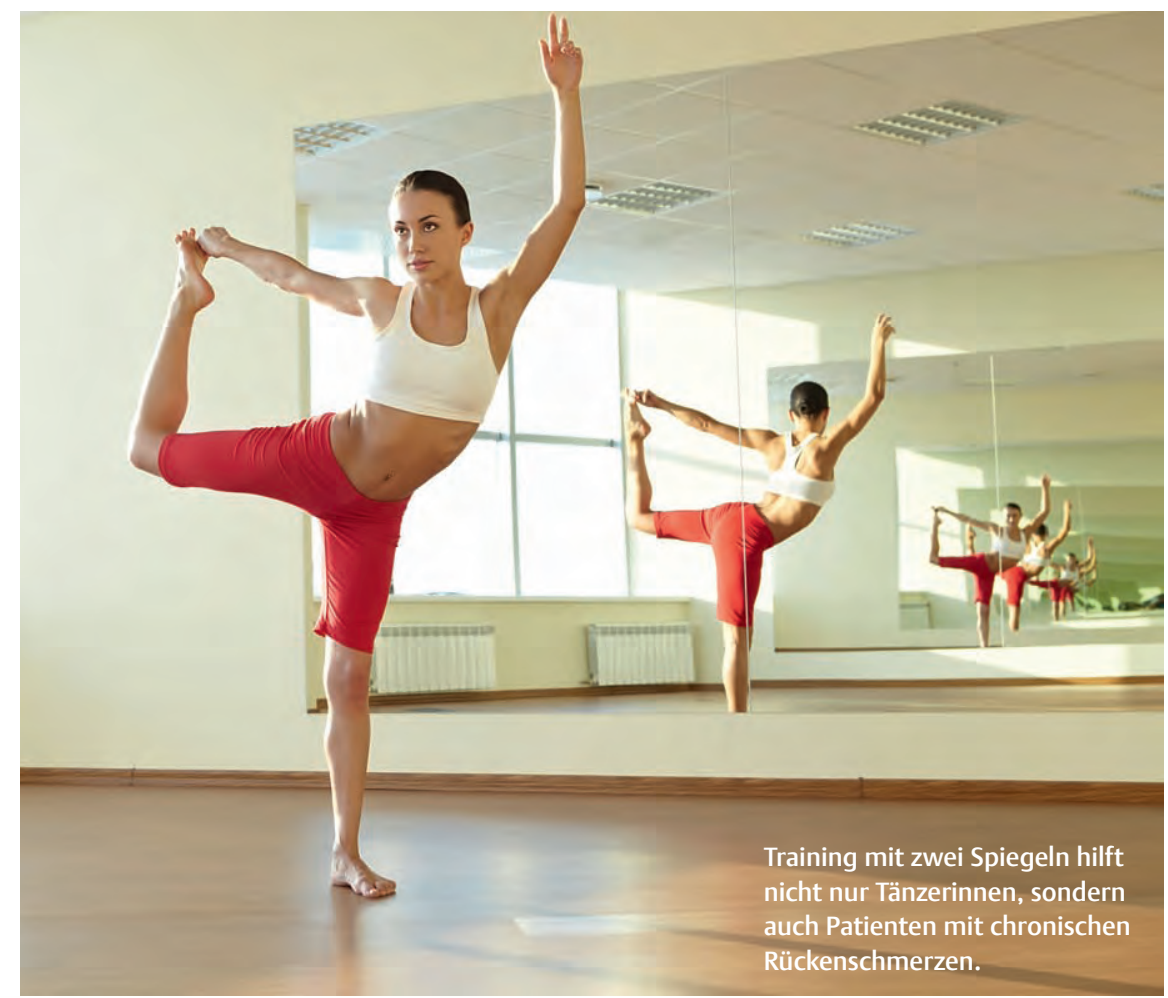

\section{CHRONISCHE RÜCKENSCHMERZEN}

\section{Therapie mit Spiegelbild}

- Spiegeltherapie kennt man bislang überwiegend zur Therapie bei Menschen mit CRPS (Complex Regional Pain Syndrome) oder mit Hemiparese. Ein Team australischer Wissenschaftler testete jetzt, welchen Effekt eine Therapie mit Spiegeln bei Menschen mit chronischen Rückenschmerzen hat - mit bemerkenswerten Ergebnissen.

Für ihre Studie rekrutierten die Forscher 25 Patienten, die seit mindestens sechs Monaten unter unspezifischen Rückenschmerzen und zum Zeitpunkt der Studie zumindest unter moderaten Schmerzen litten. Alle Patienten erhielten das gleiche Übungsprogramm mit aktiven Flex- und Extensionsübungen für die LWS. Dabei standen sie zwischen zwei Spiegeln, die so angeordnet waren, dass die Probanden ihren Rücken sehen konnten. Die Übungen führten die Teilnehmer jeweils zweimal durch: Einmal waren die Spiegel abge- hängt, sodass der Blick auf den Rücken verdeckt war, einmal waren die Spiegel offen. Zwischen den beiden Sätzen legten sich die Teilnehmer für fünf Minuten auf eine Bank. In welchem der zwei Settings die Teilnehmer zuerst übten, entschied das Los. Jeweils nach dem Übungsprogramm bewerteten die Probanden die Stärke ihrer Schmerzen.

Es stellte sich heraus, dass die Schmerzintensität nach dem Üben mit freiem Blick auf den Rücken signifikant geringer war als nach dem Training ohne Sicht - egal, in welchem Setting die Teilnehmer begonnen hatten. Die Zeit, bis sich die Schmerzen verringert hatten, war mit Spiegelbild ebenfalls signifikant geringer. Nun diskutieren die Forscher, ob der Effekt eher neurophysiologisch oder psychologisch begründet ist.

josc

Clin J Pain 2012; 28: $602-608$
ZITAT

„EIN MANGEL AN PHANTASIE BEDEUTET DEN TOD DER WISSENSCHAFT."

Johannes Kepler

\section{MORBUS PARKINSON \\ Am Anfang ist es oft „dringend“}

Menschen mit Morbus Parkinson leiden aufgrund ihrer Erkrankung auch unter vielen Symptomen, die nicht den klassischen motorischen Zeichen zuzuordnen sind. Wissenschaftler aus Großbritannien wollten nun wissen, welche dieser „nichtmotorischen“ Symptome bereits zu Beginn der Krankheit bestehen.

Dazu untersuchten sie 159 Patienten, bei denen Morbus Parkinson erst vor kurzem diagnostiziert worden war, sowie 99 Gesunde. Sie fanden heraus, dass die Erkrankten signifikant häufiger unter nichtmotorischen Symptomen litten. Die am häufigsten auftretenden waren: übermäßiger Speichelfluss, Vergesslichkeit, spontaner, starker Urindrang, verminderter Geruchssinn sowie Verstopfung. josc Neurology 2013; 80: 276-281

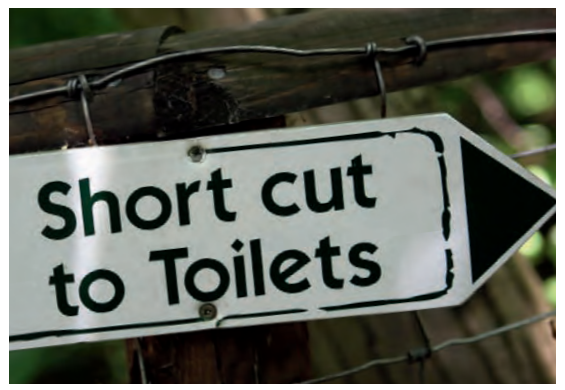




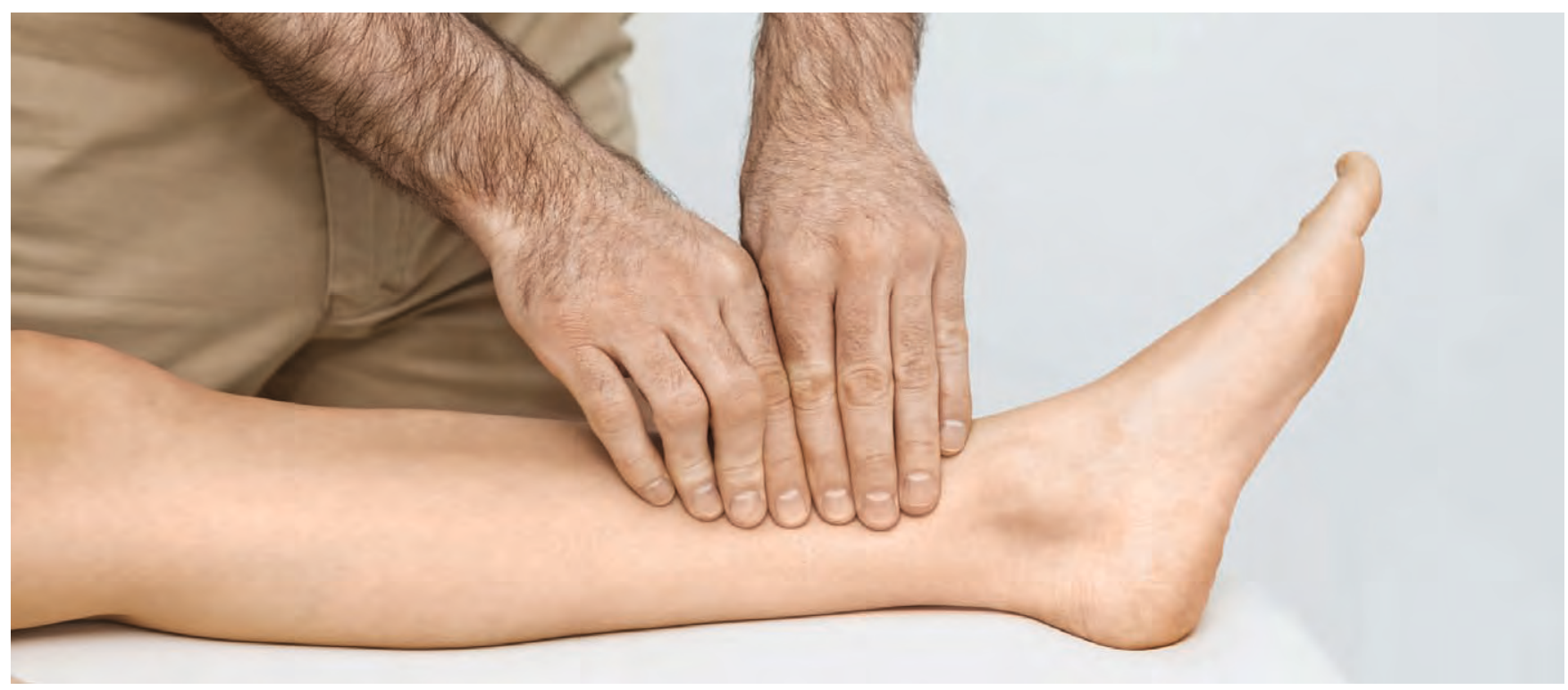

MEDIALES TIBIAKANTENSYNDROM

\section{Risiko mit Palpationstest erkennen}

Ein mediales Schienbeinkantensyndrom auch unter dem Begriff „Shin Splints“ bekannt - ist eine Überlastung der Unterschenkelmuskulatur, von der häufig Laufsportler betroffen sind. Wissenschaftler konnten nun mittels zweier Palpationstests recht zuverlässig vorhersagen, welcher der in ihrer Studie untersuchten Soldaten in den folgenden Monaten Beschwerden entwickeln wird.

384 australische Soldaten nahmen an der Studie teil, rund ein Drittel waren Frauen. Beim ersten Test palpierte der Untersucher beidseits die unteren zwei Drittel der Tibia einschließlich der posteromedialen Kante und der Muskulatur. Konnte er dabei mit einer Kraft, die etwa der beim Ausdrücken eines Schwammes entspricht, Schmerzen auslösen, galt der Test als positiv. Das war bei $20 \%$ der Teilnehmer der Fall. Bei der zweiten Untersuchung wurde geprüft, ob die Probanden Ödeme im Schienbeinbereich hatten. Dies traf auf $3 \%$ aller Probanden zu.

$15 \%$ der Teilnehmer entwickelten in den folgenden Monaten ein Tibiakantensyndrom - die größte Zeitspanne betrug 490 Tage. Personen mit einem positiven Palpationstest hat- ten ein etwa viereinhalbfach höheres Erkrankungsrisiko, wobei die Vorhersagekraft bei Männern mehr als doppelt so hoch war. Bei einem Schienbeinödem erhöhte sich das Risiko für ein Tibiakantensyndrom sogar um den Faktor 76 - lediglich einer von elf Probanden mit Ödem blieb von den Schmerzen verschont. Insgesamt lag das Erkrankungsrisiko für Frauen dreimal über dem von Männern. josc

BrJ Sports Med 2012; doi: 10.1136/ bjsports-2012-090409

\section{SCHLAGANFALL}

\section{Schnellkrafttraining verbessert Kontraktionszeit}

- Menschen nach einem Schlaganfall haben häufig Schwierigkeiten, Muskelkontraktionen und damit Bewegungen schnell auszuführen. Daher testeten Vicky Gray und ihre Kollegen aus London und Vancouver, welchen Effekt ein einmaliges Schnellkrafttraining bei diesen Patienten hat.

32 Patienten und 32 Gesunde führten einmalig 50 schnelle Kniebeugen und 50 schnelle Wiederholungen einer Steppübung durch. Bei den Squats „entriegelten“ die Probanden ihre Kniegelenke und fingen die Bewegung bei circa $30^{\circ}$ Kniegelenkflexion ab. Für die Stepp- übung neigten sie den Körper nach vorne, bis sie das Gleichgewicht verloren, machten dann einen Schritt aus dem Stand nach vorne, um sich abzufangen, und drückten sich anschließend direkt in den Zweibeinstand zurück. Mithilfe eines Elektromyogramms überprüften die Forscher die Kontraktionszeiten am Anfang und am Ende des Trainings. Sie stellten fest, dass sich die Kontraktionszeit in den übungsrelevanten Muskeln des paretischen Beines deutlich verbessert hatte. Im nichtparetischen Bein und bei Gesunden blieb sie hingegen nahezu kons- 
SCHLAGANFALL

\section{Erst kräftigen, dann auf- gabenspezifisch trainieren}

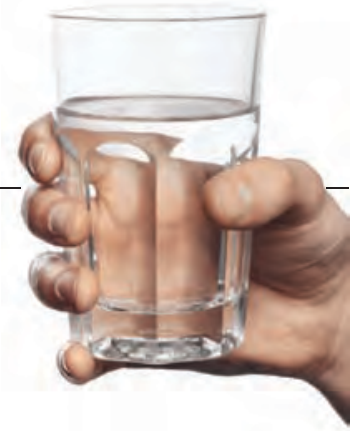

Ein aufgabenspezifisches Training normalisiert Bewegungen, fördert Kompensationen und steigert so die Armfunktion von Patienten nach Schlaganfall. Ein Krafttraining hingegen normalisiert die Muskelfunktion. Kombiniert man beide Trainingsmethoden, sind die Erfolge für den Patienten besonders gut. Das fanden amerikanische Forscher heraus.

14 Patienten mit Hemiparese nach einem Schlaganfall nahmen an einer 20 Wochen dauernden Übungsserie teil. Sieben Patienten begannen mit Krafttraining, sieben mit funktionellem Training. Die Einheiten dauerten jeweils 90 Minuten und fanden dreimal pro Woche statt. Nach der Hälfe der Zeit wechselten die
Probanden die Intervention. Vor und nach der jeweiligen Übungsphase untersuchten die Forscher die kinetischen Bewegungsabläufe und verschiedene klinisch-funktionelle Parameter.

Sie stellten fest, dass beide Interventionen die Bewegungskoordination der Patienten verbesserten. Das funktionelle Training förderte zusätzlich kompensatorische Bewegungsmuster, das Krafttraining hingegen eine normale Muskelfunktion. Besonders gute Effekte erzielten Teilnehmer, die zuerst ihre Kraft trainiert und dann aufgabenspezifisch geübt hatten.

hoth Neurorehabil Neural Repair 2012; 26: $842-854$

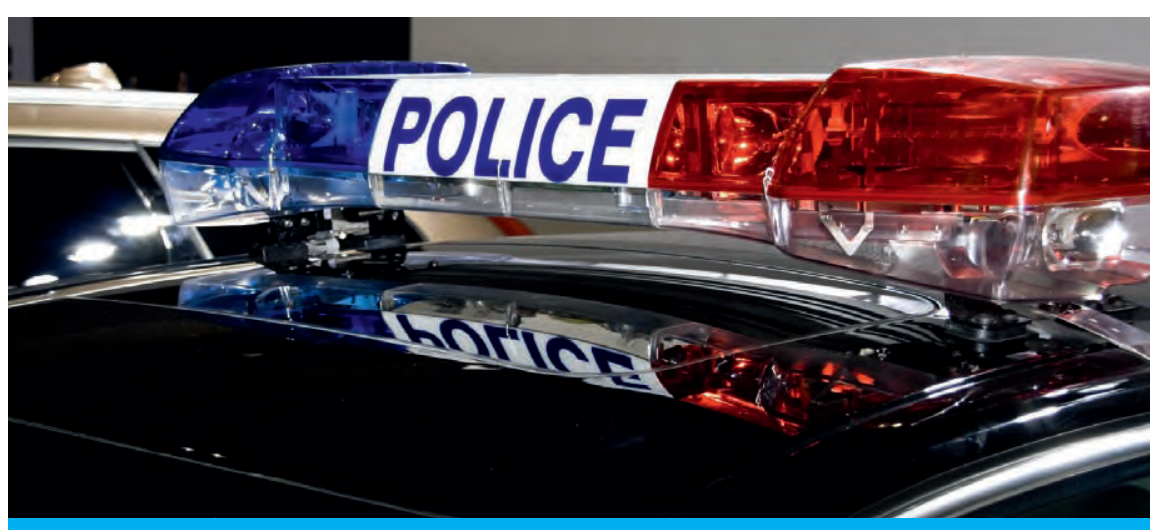

AKTUTBEHANDLUNG NACH TRAUMA

\section{Lieber POLICE statt PECH?}

Das Akronym PECH (Pause-Eis-Kompression-Hochlagern) kennt man im Englischen unter PRICE (Protection-Rest-IceCompression-Elevation). Schon seit vielen Jahren wird dieses Mantra gepredigt, vor allem bei akuten Sportverletzungen. Der Brite Chris Bleakley vom Sport and Exercise Sciences Research Institute in Ulster, Großbritannien, und zwei seiner Kollegen schlugen nun aufgrund der veränderten Studienlage vor, dies zu ändern - in POLICE.

In ihrem Editorial im British Journal of Sports Medicine betonen sie, neue Studien haben gezeigt, dass „Rest“ (Pause), also ein längerer Entlastungszeitraum, kontraproduktiv für eine optimale Heilung ist. Stattdessen sei es wichtig, den Betroffenen ein optimales Gleichgewicht von Be- und Entlastung zugutekommen zu lassen. Aus diesem Grund wählten sie das Akronym POLICE (Protection-Optimal Loading-IceCompression-Elevation). Dies solle zudem nicht nur als eine einfache „Formel“ gelten, sondern Kliniker daran erinnern, sich neue und effektive Strategien für eine optimale posttraumatische Belastung der betroffenen Struktur auszudenken. 

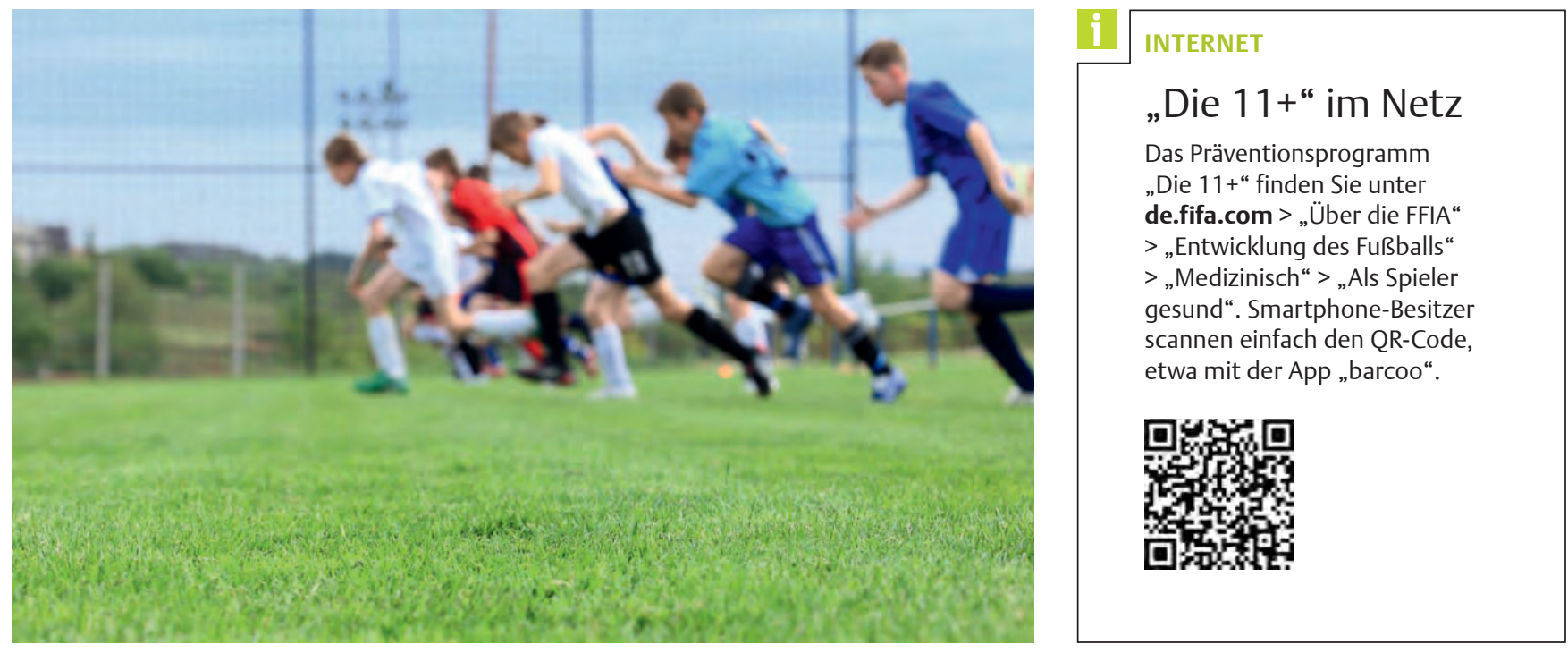

FUSSBALLVERLETZUNGEN

\section{Präventionsprogramm „Die 11+“ möglicherweise ungenügend}

— „Die 11+“ ist ein Aufwärmprogramm, das Fußballer ab dem 14. Lebensjahr laut Empfehlung des Weltfußballverbands FIFA regelmäBig durchführen sollten, um Verletzungen vorzubeugen. Es besteht aus verschiedenen Lauf-, Stabilisations- und Kraftübungen. Nun stellt eine niederländische Studie den Effekt dieses Programms in Frage.
23 Amateurfußballteams mit insgesamt 455 Spielern nahmen an der Untersuchung teil. Die Forscher teilten sie per Zufall in zwei Gruppen ein. Die eine führte während einer Saison regelmäßig das etwa 15 Minuten dauernde Präventionsprogramm durch, die andere nicht. Das ernüchternde Ergebnis: Die Zahl der verletzten Spieler sowie die Schwere

der Verletzungen waren in beiden Gruppen praktisch gleich. Der einzige Unterschied: Die Interventionsgruppe verletzte sich signifikant seltener am Kniegelenk.

josc

Br J Sports Med 2012; doi: 10.1136/ bjsports-2012-091277

\section{ACHILLODYNIE}

\section{Lasertherapie überflüssig?}

- Bei der Therapie einer chronischen Achillodynie gilt exzentrisches Training als eine der effektivsten Methoden. Nun prüften Wissenschaftler aus Neuseeland, ob es sich lohnt, die malade Achillessehne zusätzlich mit einer sogenannten Low-Level-Lasertherapie (LLLT) zu behandeln.

Die 40 an der Studie teilnehmenden Patienten wurden per Zufall in zwei Gruppen aufgeteilt. Die eine Hälfte trainierte zwölf Wochen exzentrisch und erhielt in den ersten vier Wochen zusätzlich dreimal pro Woche Lasertherapie. Die Einstellungen des Lasers passten die Wissenschaftler anhand der World Association for Laser Therapy an. Die restlichen Teilnehmer trainierten gleich, bekamen jedoch lediglich eine Schein-Laserbehandlung. Un- tersucht wurden alle Probanden vor der Studie sowie nach vier, zwölf und 52 Wochen. Als primären Outcome-Parameter verwendeten die Wissenschaftler den VISA-A-Fragebogen (Victorian Institute of Sports Assessment Achilles Questionnaire).

Die Ergebnisse: Während das exzentrische Training die Schmerzen der Probanden sowie deren Scores auf dem VISA-A deutlich verbesserten, zeigte die LLLT zu keinem Zeitpunkt einen Effekt. Im Gegenteil: Die Ergebnisse des VISA-A waren bei den Probanden mit ScheinLaserbehandlung nach vier Wochen sogar signifikant besser als bei denjenigen, welche eine „echte“ Laserbehandlung erhalten hatten.

josc

Arch Phys Med Rehabil 2012; 93: 733-739

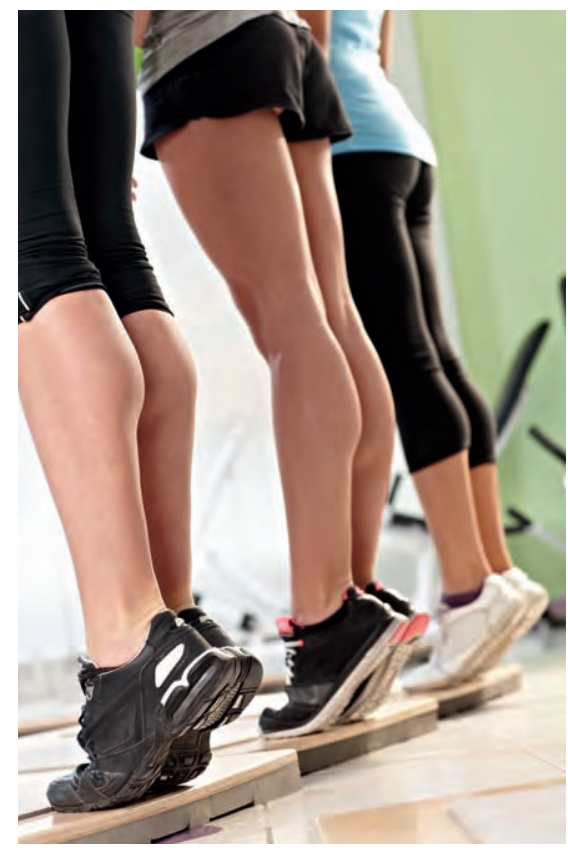




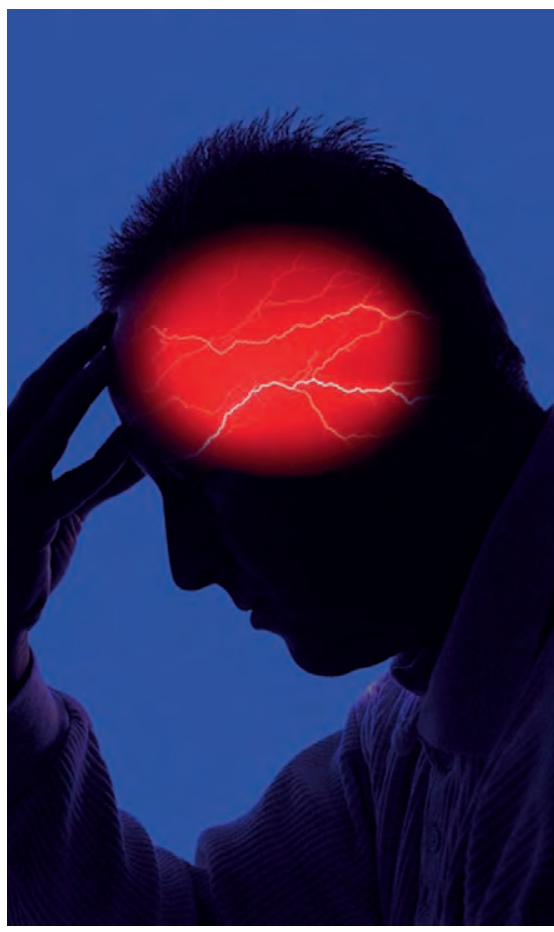

MIGRÄNE

\section{Erkrankungsverhältnis}

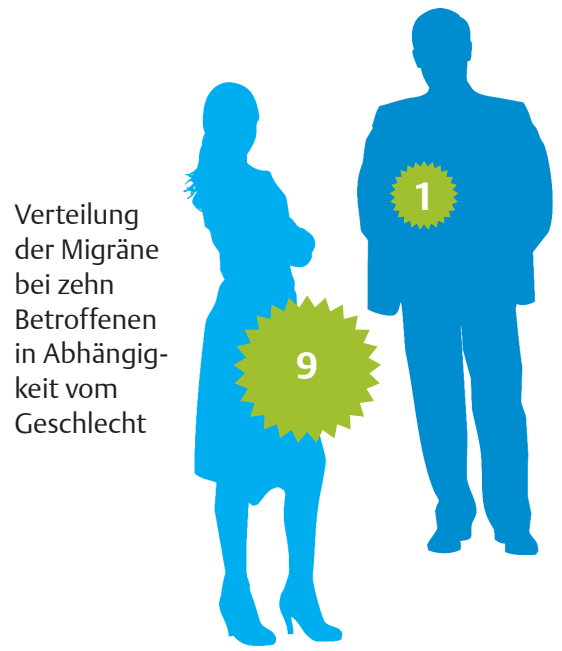

Nervenheilkunde 2007; 26: 186-199

\section{PRIMÄRE KOPFSCHMERZEN}

\section{Muskuloskeletale Komponenten prüfen}

- Im Gegensatz zu beispielsweise zervikogenen Kopfschmerzen, welche eine Auswirkung einer HWS-Problematik darstellen, zählen Spannungskopfschmerz und Migräne ohne Aura zu den primären Kopfschmerzerkrankungen - der Schmerz selbst ist also die Erkrankung. In mehreren Studien erkannten Wissenschaftler jedoch, dass viele Menschen mit Migräne auch Dysfunktionen in den hochzervikalen Segmenten haben. Der australische Kopfschmerzexperte Dean Watson wollte daher gemeinsam mit einem Kollegen herausfinden, ob sich die Symptome von Menschen mit Migräne oder Spannungskopfschmerzen auch durch eine manuelle Untersuchung der HWS reproduzieren lassen.

Sie schlossen 34 Patienten (20 mit Migräne, 14 mit Spannungskopfschmerzen) sowie 14 Gesunde in ihre Studie ein. Die Untersuchung der Patienten fand in einer schmerzfreien Episode statt. Der Untersucher wählte die Seite, auf der er den gehaltenen manuellen Druck applizierte, anhand der Symptome aus: $\mathrm{Er}$ entschied sich entweder für die schmerzhafte Seite (bei unilateralen Symptomen), die Seite, auf der die Kopfschmerzen am häufigsten auftraten (bei Symptomen, die die Seite wechselten), oder die Seite, in deren Richtung der Processus spinosus des Axis laut seines Palpationsbefundes rotiert war. Bei der Kontrollgruppe wurde die Seite per Zufall ausgewählt. Die beiden Techniken, die der Untersucher anschließend applizierte, waren:

> ein gehaltener Druck auf den hinteren Atlasbogen

$>$ ein gehaltener Druck auf den Proc. articularis von $\mathrm{C} 2$

Bei allen Patienten mit Spannungskopfschmerz und bei 19 von 20 Patienten mit Migräne lösten die Techniken den Schmerz aus, der auch bei einer Attacke auftritt. Beide Techniken verursachten beim jeweiligen Teilnehmer immer die gleichen Symptome, sowohl hinsichtlich der Lokalisation als auch der Intensität.

Bei Menschen mit Migräne oder Spannungskopfschmerzen könnten somit nozizeptive zervikale Afferenzen bislang zu wenig Beachtung gefunden haben. Die Autoren empfehlen daher auch bei diesen Patienten, muskuloskeletale Komponenten in die Untersuchung mit einzubeziehen.

josc Headache 2012; 52: 1226-1235 


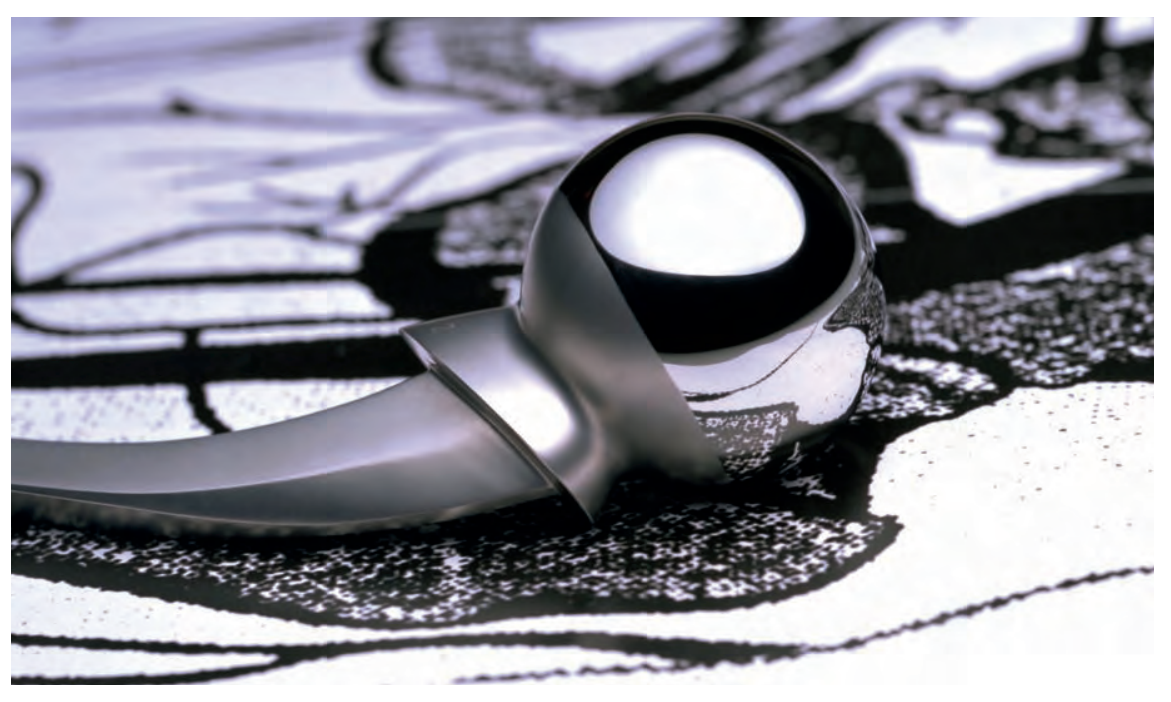

TOTALENDOPROTHESEN

\section{TEPs erhöhen Infarktrisiko}

- Patienten, die eine Knie- oder Hüftgelenkendoprothese erhalten, haben anschließend ein deutlich erhöhtes Risiko, einen Herzinfarkt zu erleiden. Das berichten Wissenschaftler aus den Niederlanden.

Sie betrachteten retrospektiv die Daten von 66.524 Patienten nach Hüft- und 28.703 Patienten nach Knie-TEP-OP und verglichen sie mit denen von 286.165 nicht operierten Kontrollpersonen. Die Operierten waren im Schnitt knapp 70 Jahre alt. In den ersten beiden Wochen nach Hüft-TEP-OP war das Infarktrisiko rund 25 Mal höher als bei der Kontrollgruppe, bis zur sechsten Woche noch rund fünfmal. Bei den Patienten mit Knie-TEP erhöhte sich das Risiko in den ersten beiden
Wochen um den Faktor 30, bereits nach zwei Wochen hatte es sich jedoch dem der nicht operierten Gruppe angeglichen.

Vor allem nach Hüftgelenkendoprothese waren hochbetagte Patienten besonders infarktgefährdet, diejenigen unter 60 unterschieden sich hinsichtlich des Risikos dagegen nicht von den Kontrollpersonen. Weitere Faktoren, welche die Infarktwahrscheinlichkeit nach Hüft-TEP-OP erhöhten, waren: männliches Geschlecht, Herzinsuffizienz, früherer Infarkt, zerebrovaskuläre Erkrankungen und die Einnahme von Medikamenten wie Betablocker und Diuretika.

josc Arch Intern Med 2012; 172: 1229-1235
HÜFT- UND KNIEGELENKARTHROSE

\section{Vorsorge und Reha}

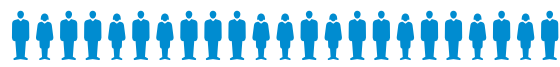

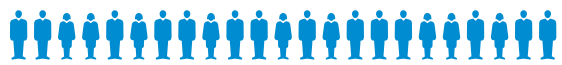

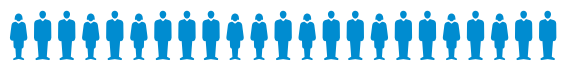

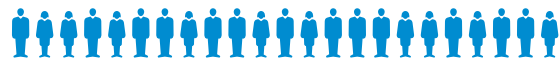

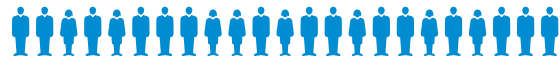

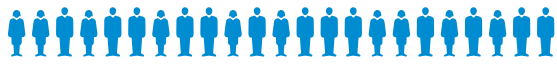

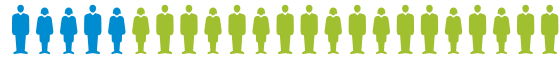
thinhthinthinth

\section{1,6 Mio. Millionen Patienten insgesamt in Vorsorge- oder Rehaeinrichtung,}

davon $\mathbf{2 1 6 . 0 0 0}$ mit Hüft- oder Kniegelenkarthrose (ca. 13\%),

davon:

11 $62 \%$ zwischen 65 und 84 Jahre

33\% zwischen 45 und 64 Jahre

$3 \%$ über 85 Jahre

Altersstruktur der Patienten, die 2011 wegen einer Hüft- oder Kniegelenkarthrose vollstationär in einer Vorsorge- oder Rehaklinik aufgenommen waren

Pressemeldung des Statistischen Bundesamts; 2013 